【研究简报】

\title{
非线性 $\mathbf{H}_{\infty}$ 控制的粘性解方法
}

\author{
洪奕光永绍鹏可梅生伟 秦化淑 \\ ( I 中国科学院系统科学研究所, 北京 100080; 香港大学数学系, 香港)
}

\section{关键词 非线性 $\boldsymbol{H}_{\infty}$ 粘性解 千扰抑制}

考虑系统:

$$
\begin{aligned}
& \dot{x}=F(x, u, w), \\
& i z=Z(x, u, w),
\end{aligned}
$$

这里, $F, Z \in \mathrm{C}^{1}\left(z^{n}\right), F(0,0,0)=0, Z(0,0,0)=0, x \in Z^{n}$ 状态变量, $u \in U \in Z^{n}$ 控制变 量, $w \in W \in Z$ 外界干扰, $z \in \Xi$ 调节输出变量. $U$ 和 $W$ 是紧集.

定义 非线性 $H_{x}$ 问题 (或非线性干扰抑制) 就是要对系统 (1.1) 寻找最小的正数 $\gamma^{*}, \forall \gamma$ $>\gamma^{*}$, 总可设计一个控制器使得

1) 初始值 $x(0)=0$ 时有

$$
\int_{0}^{t_{0}} z^{\mathrm{T}} z \mathrm{~d} s \leqslant \gamma^{2} \int_{0}^{t_{\tilde{b}}} w^{\mathrm{T}} w \cdot \mathrm{d} s, \quad \forall T_{0}>0 ;
$$

2) 内部稳定性: 当 $w^{\prime}=0$ 时, 该控制器能使相应的闭环系统渐近稳定.

这里考虑两种控制器: 状态反馈控制器 $u=u(x)$ 或全信息反馈控制器 $u=u(x, w)$ (如 果干扰可被测量).

考虑到非线性 $\mathrm{H}_{\infty}$, 包括两个层次的问题：（i）如何选取尽可能小的且代表干扰抑制性 能的 $L_{2}$ 增益 $\gamma$; (ii) 对已经给定的增益, 如何给出合适的控制器.

利用二人零和微分对策理论, 可分析上述问题. 只要能找到一个正定函数 $V$, 则(2)式可 被得到, 且 $V(0)=0, \forall t>t_{0} \geqslant 0$ 使下面不等式成立:

$$
V\left(x\left(t_{0}\right)\right) \geqslant \inf _{u} \sup _{u}\left(V(x(t))+\int_{t_{0}}^{t}\left(z^{\mathrm{T}} z-\gamma^{2} w^{\mathrm{T}} w\right) \mathrm{d} s\right),
$$

或利用 Hamilton 函数 $H(x, p, u, w)=p F+z^{\mathrm{T}} z-\gamma^{2} w^{\mathrm{T}} w^{\prime}$ 可得 Hamilton-Jacobi-Issac 微分 不等式:

$$
H \cdot(x, \mathrm{D} V)=\inf _{u} \sup _{u}\left(\mathrm{D} V \cdot F+z^{\mathrm{T}} z-\gamma^{2} w^{\mathrm{T}} w\right) \leqslant 0,
$$

其中 D 表示关于 $x$ 的 Dini 导数.

通常为了解此问题, 还不得不假定光滑函数 $V$ 是存在的, 这在某些时候会影响控制器的 设计性能. 本文将利用粘性解讨论 $V$ 是不光滑的情况. 其实, 在偏微分方程和不等式中已有 粘性解的研究, 并且也已用到非线性 (随机) 最优控制中 [1]. 从已有的理论中可知, 当 $L_{2}$ 增益 $\gamma$ 小到一定时, 微分不等式 (或微分等式) (4) 将没有光滑解, 但是其粘性解可能还是存在 的[2]. 实际上, 在一个具体的求解不等式的过程中, 同时也可考虑能否进一步使 $\gamma$ 的数值变 小而不影响不等式解的存在性. 另外, 粘性解的应用还可以扩大解的有效适用范围.

一般地，考虑 $\Xi^{n}$ 中开集 $Q$ 上的一阶偏微分不等式

$$
P(\approx, V(z), D V(z)) \leqslant 0,
$$


这里, $z \in \mathrm{Q}, P: \mathrm{Q} \times \vec{Z} \times \mathrm{B}^{n} \rightarrow \vec{B}, V(\cdot)$ 局部有界. 我们称局部有界函数 $V(\cdot)$ 是 $(5)$ 式的一个粘 性解, 如果对任何 $\varphi(\cdot) \in C^{1}(Q)$, 只要 $V \cdot(\cdot)-\varphi(\cdot)$ 在 $: \infty \in \mathrm{Q}$ 达到局部极小, 则有

$$
P\left(z_{0}, V\left(z_{0}\right), \mathrm{D} \varphi\left(Z_{0}\right)\right) \leqslant 0 .
$$

其中 $V$, 是 $V$ 的下半连续包, 即

$$
V \cdot(x)=\liminf _{z \rightarrow x} V(z) \text {. }
$$

定理 1 如果一个局部有界的半正定函数 $V$ 满足 (3) 式, 那么它的下半连续包 $V .(x)$ 也 满足 (3) 式, 即若 $V$ 是 (3)式的 (粘性) 解, 则 $V$, 也是.

证 记 $U$ 是容许控制集, $\beta(u, w, t) x_{0}$ 是在干扰 $w$ 和控制 $u$ 下以 $x_{0}$ 为初值的流算子, $L(\cdot)=\gamma^{2} w^{\mathrm{T}} w-z^{\mathrm{T}} z$. 固定 $t \geqslant t_{10}$ 和 $x\left(t_{0}\right)=x_{0} \in z^{n} . \forall w$, 取最优 $u=u .(x, w) \in U$ 使 (3) 式右边局部最小, 则由(3)式可得

$$
V\left(x_{0}\right) \geqslant V\left(\beta(u,, w, t) x_{0}\right)-\int_{t_{0}}^{t} L\left(\beta(u,, w, r) x_{0}\right) \mathrm{d} r,
$$

选一序列: $x_{i}:\left.\right|_{i=1} ^{\infty}$ 使得 $x_{0}=\lim _{i \infty} x_{i}$ 和 $V .\left(x_{i}\right)=\lim _{i \rightarrow \infty} V\left(x_{i}\right)$. 因为 $V \geqslant V \ldots$, 由 (3) 式可得

$$
\begin{array}{r}
V\left(x_{i}\right) \geqslant V\left(\beta(u,, w, t) x_{i}\right)-\int_{t_{0}}^{t} L\left(\beta(u,, w, r) x_{0}\right) \mathrm{d} r \geqslant \\
V .\left(\beta(u, \ldots, t) x_{i}\right)-\int_{t_{0}}^{t} L\left(\beta(u, w, r) x_{0}\right) \mathrm{d} r .
\end{array}
$$

令 $i \rightarrow \infty$, 可知

$$
V .\left(x_{0}\right) \geqslant \liminf _{x \rightarrow \infty} V \cdot\left(\beta(u,, w, t) x_{t}\right)-\int_{t_{0}}^{t} L\left(\beta(u, w, r) x_{0}\right) \mathrm{d} r .
$$

因 $\forall w(7)$ 式都成立, 故有

$$
V .\left(x_{0}\right) \geqslant \sup _{x}\left|V .-\int_{t_{u}}^{t} L\left(\beta(u,, w, r) x_{0}\right) \mathrm{d} r\right| \geqslant \inf _{u} \sup _{w}\left|V .-\int_{t_{0}}^{t} L\left(\beta(u, w, r) x_{0}\right) \mathrm{d} r\right| .
$$

故命题得证.

其实, 对(4)式可给出类似的结果. 为此先证明:

定理 2 若 $V$ 满足 (3) 式, $V$ 就满足 (4)式.

证 由于 $V$ 不一定光滑, 所以不能直接求导.

取 $\varphi \in \mathrm{C}^{\mathrm{l}}(\mathrm{Q})$ ，假定 $V_{*}-\varphi$ 在 $x_{0} \in \mathbb{Z}^{n}$ 达到局部极小，我们将证

$$
H\left(x_{0}, \mathrm{D} \varphi\left(x_{0}\right)\right)=\inf _{u} \sup _{u}\left(\mathrm{D} \varphi\left(x_{0}\right) F\left(x_{0}\right)+L\left(x_{0}\right)\right) \leqslant 0,
$$

对 $t \geqslant t_{0}$, 取 $w \in W$ 为常值, 控制 $u=u .(x, w) \in U$ 为最优解. 记 $x(t)=\beta(u, w, t) x_{0}$.

对 $t \geqslant t_{0}$ 使 $t-t_{0}$ 充分小, 我们有(因 $x_{0}$ 是极小点)

$$
V \cdot\left(x_{0}\right)-V \cdot(x(t)) \leqslant \varphi\left(x_{0}\right)-\varphi(x(t)) .
$$

由定理 1 , 有

$$
V \cdot\left(x_{0}\right)-V \cdot(x(t)) \geqslant \int_{t_{0}}^{t} L(x(r)) \mathrm{d} r
$$

于是有

$$
\frac{\varphi(x(t))-\varphi\left(x_{0}\right)}{t-t_{0}}+\frac{1}{t-t_{0}} \int_{t_{0}}^{t} L(x(r)) \mathrm{d} r \leqslant 0 .
$$


令 $t \rightarrow t_{0}$, 就可得

$$
\mathrm{D} \varphi\left(x_{0}\right) f\left(x_{0}\right)+L\left(x_{0}\right) \leqslant 0,
$$

因此(4)式成立, 故得证.

由于由 (4)式可能推出 (3)式, 也就是说上述定理表明即使在粘性解意义下 (3) 与 (4)式也 是等价的.

注意在上述讨论中, 控制器选取的形式为 $u=u *(x, w)$. 事实上, 如果假定鞍点条件成 立, 即 $H(x, \mathrm{D} V, u, w)$ 有局部唯一的鞍点 $\left(u, w^{\prime}\right)$ 对每一个 $x$, 也就是说, 存在局部唯一的 $u_{*}(x)$ 和 $w_{*}(x)$, 使得

$$
H\left(x, \mathrm{D} V, u_{*}, w^{\prime}\right) \leqslant H\left(x, \mathrm{D} V, u_{*}, w_{*}\right) \leqslant H\left(x, \mathrm{D} V, u_{,} w_{*}\right),
$$

这时控制器形式为 $u_{*}(x)$ 与 $w$ 无关, 是状态控制器. 根据文献 [3], 假定 $\frac{\partial Z}{\partial w}=0, \frac{\partial Z}{\partial u}(0,0)$ 是行满秩的, 就能保证上述二人零和对策问题满足局部唯一的鞍点条件.

至此, 我们只考虑了如何使 (2) 式成立, 下面将进一步讨论系统 (1) 的内部稳定性. 为此 给出零状态可检测 ( $w=0$ 时) 的假定: 即 $w=0$ 时, 由 $z=0$ 可推出 $x \rightarrow 0$.

定理 3 假定系统 (1) 满足上面零状态可检测假设, 且 $V$ 是定义在原点邻域满足 (4) 式的 局部有界非负粘性解, 而且它在原点处正定连续并 $V(0)=0$. 那么控制律 $u=u *(x, w)$ (或 鞍点条件成立时的 $u=u .(x))$ 就可使系统 (1) 满足 (2) 式且无干扰时内部稳定性.

证 注意由以前的讨论易知(2)式也成立.

当 $w=0$ 时, 有

$$
\left.V(x(t))\right|_{u=u} \cdot-V\left(x_{0}\right) \leqslant-\int_{t_{0}}^{t}\|Z\|^{2} \mathrm{~d} s \leqslant 0, \quad x(0)=x_{0} .
$$

由 $V$ 的正定性可知: 存在一个严格增的连续函数 $b:[0, \infty), b(0)=0$, 且 $V(x) \geqslant b(|x|)$. 因此有 $V .(x) \geqslant b(|x|)$. 对任给 $\varepsilon>0$, 由 $V$ 在原点的连续性, 存在 $\delta>0$, 使得 $|x|<\delta$ 时 $V .(x)<b(\varepsilon)$. 由定理 1 和 2 , 可知 $V_{*}$ 也满足 (9)式. 于是有

$$
b(|x(t)|) \leqslant V_{*}(x(t)) \leqslant V_{*}\left(x_{0}\right)<b(\varepsilon), \quad t \geqslant 0,
$$

只要 $\left|x_{0}\right|<0$, 因此 $|x(t)|<\varepsilon$. 故稳定性可证.

下面证明渐近稳定性. 考虑对充分小初始值的 $\omega$-极限集 $\Gamma$. 记 $x(t)=\beta(u,, 0, t) x_{0}$.

$$
\lim _{t \rightarrow \infty} V_{*}(x(t))=c \geqslant 0,
$$

当 $x \in \Gamma$ 且 $V,(x)=c$ 时, 因为 (9)式, $V,(\beta(u,, 0, t) \bar{x})$ 不会大于 $c$. 另外, 既然 $\bar{x}$ 是极 限集上的点, 那么 $\forall i$ 就有 $t_{i}$ 使 $\left|x_{i}-\bar{x}\right| \leqslant 1 / i$. 其中 $x_{i}=\beta\left(u,, 0, t_{2}\right) \bar{x}$ 及 $\lim _{1 \rightarrow \infty} t_{i}=\infty$, 因此 有 $\lim _{i \rightarrow \infty} x_{i}^{j}=x$. 注意到 $V *$ 的定义, 于是就有一序列 $x_{i}^{j}$ 使 $V .\left(x_{i}\right)=\lim _{i \rightarrow \infty} V\left(x_{i}^{j}\right)$. 再由于 $\lim _{i \rightarrow x \rightarrow x} \lim _{i} x_{i}^{j}=\bar{x}$, 可得

另外, 根据(9)式可知

$$
V *(\bar{x})=\liminf _{z \rightarrow x} V(z) \leqslant \lim _{i \rightarrow \infty} \lim _{j \rightarrow \infty} V\left(x_{i}^{j}\right)=\lim _{i \rightarrow \infty} V\left(x_{i}^{j}\right) .
$$

$$
\lim _{i \rightarrow \infty} V \cdot\left(x_{i}\right)-V \cdot(\bar{x}) \leqslant-\int_{0}^{\infty} z^{\mathrm{T}} z \leqslant 0,
$$

与 (10) 式比较、易知 $\approx(x, u, 0)=0, \forall x \in \Gamma$. 再由零可检测假定, 有 $\lim _{t \rightarrow \infty} \beta(u *, 0, t) x=0$, 因此 $c=0$. 再从 $\lim _{t \rightarrow \infty} V .(x(t))=0$ 和 $V$. 在原点附近的正定性, 可推出 $\lim _{t \rightarrow \infty} x(t)=0$. 


\title{
素特征域上的有限维 Cartan 型 Lie 超代数
}

\author{
淠永正 \\ (东北师范大学数学系, 长春 130024 )
}

\section{关链词 外代数 除䖨代数 Lie 超代数}

关于素特征域上的 Lie 超代数, 至今结果尚少. 本文构造了 F 上的无限维 Cartan 型 Lie 超代数 $X(m, n)(X=W, S, H$ 或 $K)$, 进而定义了有限维的广义 Cartan 型 Lie 超代数, 并且 讨论了它们的单性与限制性. 最后给出一个关于 $F$ 上有限维单 Lie 超代数的分类的猜想.

设 $F$ 是特征 $p>2$ 的域, $n$ 是大于 1 的正整数, $\wedge(n)$ 是 $F$ 上具有生成元 $\xi_{1}, \cdots, \xi_{n}$ 的外代 数. 若 $u=\left(i_{1}, i_{2}, \cdots, i_{r}\right)$, 其中 $1 \leqslant i_{1}<i_{2}<\cdots<i_{r} \leqslant n$, 则令 $\xi^{u}=\hat{\xi}_{i_{1}} \xi_{i}, \cdots \xi_{i_{r}},\left|\hat{\xi}^{u}\right|=r$. 设 $\bar{D}_{i}$ $=\frac{\partial}{\partial \hat{\xi}_{i}}, i=1, \cdots, n$. 仿照特征零的情形 (见文献 [1], $\S 3$ ), 可得到 $F$ 上的有限维单的 Cartan 型 Lie 超代数:

$$
\begin{aligned}
& W(n)=\sum_{i=1}^{n} a_{i} \bar{D}_{i} \mid a_{i} \in \wedge(n) \vdots, \\
& S(n)=D_{i j}(a) a \in \wedge(n), i, j=1, \cdots, n, \text {, 其中 } D_{i j}(a)=\bar{D}_{i}(a) \bar{D},+\bar{D}_{j}(a) \bar{D}_{i}, \\
& H(n)=\left\langle D_{H}\left(\xi^{u}\right)\left|\xi^{u}\right| \leqslant n-1, \text {, 其中 } D_{H}\left(\xi^{u}\right)=\sum_{i=1}^{n} \bar{D}_{i}\left(\xi^{u}\right) \bar{D}_{i},\right. \\
& \tilde{S}(n)=\left(1-\xi_{1} \xi_{2} \cdots \xi_{n}\right) D_{i j}(a) \mid a \in \wedge(n), i, j=1, \cdots, n, n \text { 为偶数. }
\end{aligned}
$$

设 $L=L_{\overline{0}} \oplus L_{\overline{1}}$ 是 $F$ 上的 Lie 超代数，如果 $L_{\overline{0}}$ 是限制 Lie 代数, 并且. $L_{\overline{0}}-$ 模 $L_{\overline{1}}$ 是限制的, 则 $L$ 是限制 Lie 超代数 (参见文献 $[2]$ ).

定理 $1 W(n), S(n), H(n)$ 与 $\tilde{S}(n)$ 是限制 Lie 超代数.

证 因为 $W(n)=\operatorname{der} \wedge(n)$, 故 $W(n)$ 是限制的. 设 $D_{i j}\left(\xi^{u}\right) \in S(n)_{\overline{0}}$. 若 $\mid \xi^{u}>2$, 直接 验证可知 $\left(D_{i j}\left(\xi^{u}\right)\right)^{2}=0$, 故 $\left(D_{i j}\left(\xi^{u}\right)\right)^{p}=0$. 若 $\xi^{u}=2$, 设 $\xi^{u}=\xi_{i} \hat{\xi}_{\text {. }}$. 则 $\left(D_{i j}\left(\xi^{u}\right)\right)^{p}=\left(\xi_{j} \bar{D}_{j}-\right.$

致谢 本工告为国家自然科学基金(批准吾: 69391901)资助项目。

\section{参考文献}

1 James M R, A partial differential inequality for dissipative nonlinear systems. System and Control Letter, 1993, 21(4):315 320

2 Lions P. Souganidis P. Differential games, optimal control and directional derivatives of viscosity solutions of Bellman' s and Issac's equations. SIAM J Control and Optim, 1985, 23(3):566 583

3 Isidori A. Kang W. $\mathrm{H}_{x}$ control via measurement feedback for general nonlinear systems. IEEE Trans AC, 1995, $40(3): 466$ $\sim 471$ 